\title{
Case Report \\ Fenofibrate: A Nonlithogenic Means of Recurrent Drug-Induced Pancreatitis
}

\author{
Thamer Kassim (D), ${ }^{1}$ Joy-Marie Hermes, ${ }^{2}$ Abdullah Abdussalam, ${ }^{3}$ \\ Ahmed Aly, ${ }^{4}$ and Subhash Chandra $\mathbb{D}^{3}$ \\ ${ }^{1}$ Department of Medicine, Division of General Internal Medicine, Creighton University, Omaha, NE, USA \\ ${ }^{2}$ Creighton University School of Medicine, Omaha, NE, USA \\ ${ }^{3}$ Department of Medicine, Division of Gastroenterology, Creighton University, Omaha, NE, USA \\ ${ }^{4}$ Department of Radiology, Creighton University, Omaha, NE, USA
}

Correspondence should be addressed to Subhash Chandra; subhash.chandra@alegent.org

Received 11 May 2018; Accepted 27 August 2018; Published 9 September 2018

Academic Editor: R. J. L. F. Loffeld

Copyright (C) 2018 Thamer Kassim et al. This is an open access article distributed under the Creative Commons Attribution License, which permits unrestricted use, distribution, and reproduction in any medium, provided the original work is properly cited.

\begin{abstract}
Medications account for a small portion of the various etiologies of acute pancreatitis. Prompt identification of drugs as the inciting factor decreases disease recurrence and unnecessary invasive diagnostic intervention. This case is a report of fenofibrateinduced acute pancreatitis including a disease recurrence with continuation of fenofibrate which subsequently resolved after drug discontinuation. The patient underwent invasive diagnostic evaluation including endoscopic ultrasound with fine needle aspiration and endoscopic retrograde cholangiopancreatography (ERCP). Based on exclusion of other disease etiologies and a positive drug rechallenge, fenofibrate fits as a class $1 \mathrm{~A}$ medication in the classification of drug-induced pancreatitis.
\end{abstract}

\section{Introduction}

The etiology of acute pancreatitis is identified in $70-90 \%$ of cases. Gallstones (up to 45\%) and alcohol use (up to $35 \%)$ overwhelmingly produce most cases. Other precipitating factors include hypertriglyceridemia, ERCP, hypercalcemia, smoking, scorpion venom, malignancy, infection, and trauma (10\% combined) [1]. The remaining $10-30 \%$ of cases are classified as idiopathic pancreatitis $[1,2]$. This percentage continues to shrink with improvement of diagnostic modalities and increased literature regarding drug-induced pancreatitis, which accounts for approximately $2 \%$ of cases $[2,3]$.

Drug-induced pancreatitis is a diagnosis fraught with difficulty. It is made when symptoms resolve with drug cessation and recur in accordance with a rechallenge of the same drug [4]. In this report, we examine a case of fenofibrate-induced recurrent pancreatitis.

\section{Case Presentation}

A 79-year-old male with a medical history of cholelithiasis, for which he underwent cholecystectomy 11 years ago, hyperlipidemia, essential hypertension, and paroxysmal atrial fibrillation was admitted for mild acute pancreatitis. His presentation included severe epigastric pain radiating to the back, lipase of $1840 \mathrm{u} / \mathrm{L}$, and no organ failure. He denied fever, chills, or body aches. The patient denied alcohol consumption and reported to have quit smoking 41 years ago. Liver chemistries, serum bilirubin, serum triglycerides, and immunoglobulin subclasses were within normal limits (Table 1). Abdominal CT scan demonstrated peripancreatic haziness consistent with noncomplicated acute pancreatitis (Figure 1). Home medications included atorvastatin for several years and the addition of $160 \mathrm{mg}$ fenofibrate six months prior to admission. These medications were held upon admission but resumed at discharge. Full medication lists on admission and at discharge are shown in (Table 2). The patient was managed with fluid resuscitation and pain control. The patient recovered well and was able to tolerate regular diet without any pain or nausea. The patient was discharged in a stable condition after three hospital admission days, and lipase level at the day of discharge was $307 \mathrm{u} / \mathrm{l}$.

Three days later, the patient returned with similar symptoms. Lipase levels were $>30,000 \mathrm{u} / \mathrm{l}$ (Table 1), and 
TABLE 1: Laboratory markers: initial admission; second admission after drug rechallenge.

\begin{tabular}{|c|c|c|c|}
\hline \multirow{2}{*}{$\begin{array}{l}\text { Laboratory test } \\
\text { Pancreatic enzymes }\end{array}$} & Initial Admission & \multirow[t]{2}{*}{ Second admission - 72 hours after discharge } & \multirow[t]{2}{*}{ Reference range } \\
\hline & & & \\
\hline Amylase (u/l) & 158 & 671 & $20-90$ \\
\hline Lipase $(\mathrm{u} / \mathrm{l})$ & $1840 \quad 307$ at discharge & $>30,000$ & 73-393 \\
\hline \multicolumn{4}{|l|}{ Basic metabolic panel } \\
\hline Glucose (mg/dl) & 101 & 75 & $70-100$ \\
\hline Sodium $(\mathrm{mmol} / \mathrm{L})$ & 136 & 138 & $135-145$ \\
\hline Potassium $(\mathrm{mmol} / \mathrm{L})$ & 4.2 & 3.6 & $3.7-5.1$ \\
\hline Chloride $(\mathrm{mmol} / \mathrm{L})$ & 104 & 102 & $96-110$ \\
\hline Carbon dioxide (mmol/L) & 24.0 & 28 & $22.0-32.0$ \\
\hline Calcium (mg/dl) & 9.0 & 8.8 & $8.5-10.5$ \\
\hline Creatinine (mg/dl) & 1.46 & 1.36 & $0.60-1.30$ \\
\hline Blood urea nitrogen mg/dl & 22 & 17 & $6-24$ \\
\hline Glomerular filtration rate $(\mathrm{mL} / \mathrm{min} / 1.73 \mathrm{~m} 2)$ & 45 & 49 & $>=90$ \\
\hline Anion Gap (mmol/L) & 12 & 12 & $<=20$ \\
\hline \multicolumn{4}{|l|}{ Hepatic function panel } \\
\hline Alanine aminotransferase $(\mathrm{u} / \mathrm{l})$ & 28 & 44 & $12-78$ \\
\hline Aspartate aminotransferase $(\mathrm{u} / \mathrm{l})$ & 33 & 37 & $10-40$ \\
\hline Alkaline Phosphatase (u/l) & 61 & 86 & $33-138$ \\
\hline Total bilirubin (mg/dl) & 1.1 & 0.5 & $0.0-1.5$ \\
\hline Total protein (gm/dl) & 7.5 & 7.4 & $6.0-8.4$ \\
\hline Albumin (gm/dl) & 3.4 & 3.3 & $3.5-5.0$ \\
\hline \multicolumn{4}{|l|}{ Immunoglobulin subclasses } \\
\hline IgG Subclass 1 (mg/dl) & 626 & & $405-1011$ \\
\hline IgG Subclass $2(\mathrm{mg} / \mathrm{dl})$ & 498 & & $169-786$ \\
\hline IgG Subclass 3 (mg/dl) & 33 & & $11-85$ \\
\hline IgG Subclass $4(\mathrm{mg} / \mathrm{dl})$ & 19 & & $3-201$ \\
\hline \multicolumn{4}{|l|}{ Other laboratory tests } \\
\hline Triglycerides (mg/dl) & 74 & & $<149$ \\
\hline White blood cell (k/ul) & 14.7 & 10.8 & $4.0-12.0$ \\
\hline Hemoglobin (gm/dl) & 15.2 & 14.1 & $13.5-17.5$ \\
\hline Platelets (k/ul) & 237 & 298 & $140-440$ \\
\hline
\end{tabular}

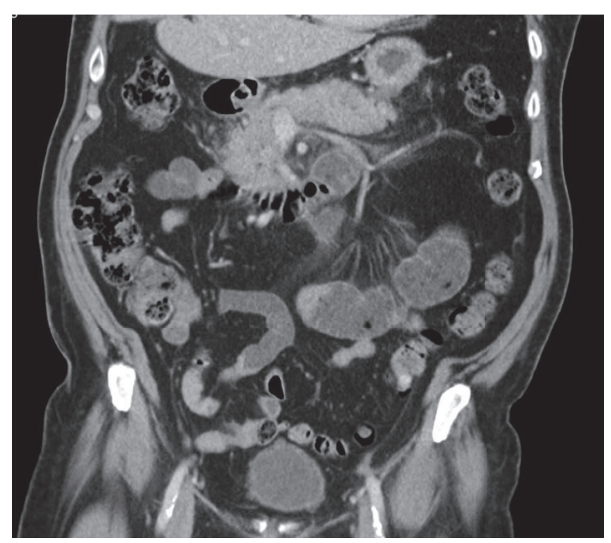

FIGURE 1: Coronal contrast enhanced CT image showing a homogenously enhancing bulky pancreas with peripancreatic haziness (arrows) with no evidence of collections consistent with noncomplicated acute interstitial pancreatitis. 


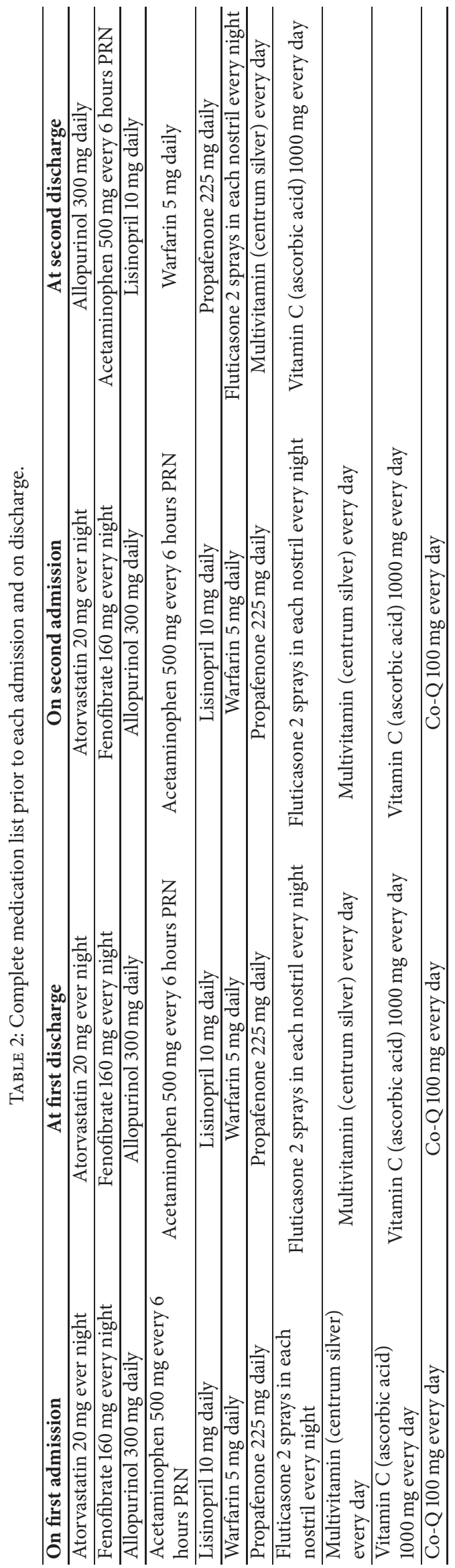




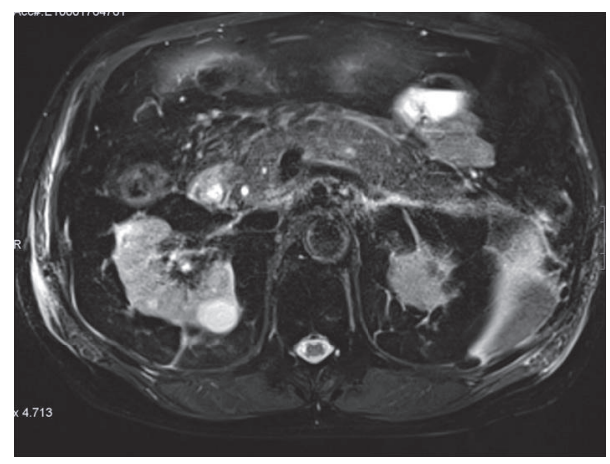

FIGURE 2: Fat suppressed T2W axial image showing both peripancreatic haziness and edema (arrows) consistent with acute interstitial pancreatitis.

magnetic resonance cholangiopancreatography showed acute interstitial edematous pancreatitis (Figure 2). The patient was treated conservatively with intravenous fluid resuscitation, pain control, and nothing per mouth until his symptoms resolved. Four days after his second admission, the patient recovered well and was discharged home in a stable condition. Fenofibrate and atorvastatin were discontinued (Table 2).

Two months later, the patient remained asymptomatic and returned for further workup to rule out an alternative cause to fenofibrate-induced pancreatitis. Endoscopic ultrasound (EUS) was done, which showed a $12 \mathrm{~mm}$ x $20 \mathrm{~mm}$ pancreatic head mass without pancreatic duct stenosis, strictures, or dilation. There were no common bile duct abnormalities. Fine needle aspiration was performed, and cytology was negative for malignant cells. Endoscopic retrograde cholangiopancreatography (ERCP) was performed to place a prophylactic pancreatic duct stent. Cholangiogram revealed a normal biliary system without dilation or strictures. The biliary tree was swept, and nothing was found. A prophylactic pancreatic duct stent was placed and a prophylactic sphincterotomy was performed. No recurrence of pancreatitis has occurred as of 6-month follow-up.

Given the course of the patient's illnesses in relation to fenofibrate usage, the timespan of drug initiation, and the fact that he had been taking atorvastatin for many years without previous signs or symptoms of acute pancreatitis, we hypothesize that his recurrent disease is probably due to the use of fenofibrate.

\section{Discussion}

Drug-induced pancreatitis accounts for $0.1 \%-2 \%$ of all cases of acute pancreatitis. Diagnosis of drug-induced pancreatitis is made when symptoms resolve with drug cessation and recur in accordance with a rechallenge of the same drug [4]. The disease is classified, from class I to IV, based on the number of cases reported, demonstration of a consistent latency, and reaction with rechallenge [5]. Three patterns of consistent latency are noted: a short latency of less than 24 hours, an intermediate latency of 24 hours to 30 days, and a long latency of greater than 30 days [5].
Typically, severity of drug-induced pancreatitis ranges from mild to moderate, but severe and even fatal cases may occur. The mechanism behind the condition remains controversial and varies between different offending medications [4]. Possible mechanisms include pancreatic duct constriction, cytotoxic effects, hypersensitivity reactions, and accumulation of toxic metabolites. Some drugs may cause pancreatitis indirectly by inducing hyperlipidemia or hypercalcemia $[4,6]$.

Lipid-modifying treatment with fibrates has been associated with the development of acute pancreatitis $[7,8]$. According to the Coronary Drug Research Project, clofibrate was associated with a 50\% increased risk of developing cholelithiasis or cholecystitis versus placebo, thus increasing the risk of acute pancreatitis [7]. Fenofibrate is believed to be less likely to induce gallstone formation while bezafibrate may raise biliary cholesterol concentration, thereby increasing the potential for gallstone formation and possibly resulting in acute pancreatitis [8]. However, there is limited data to support this theory.

Prior case reports have implicated clofibrate and bezafibrate as potential precipitating causes of acute pancreatitis $[9,10]$. The only case involving fenofibrate-associated pancreatitis was reported by McDonald et al. [1] (2002) in which a patient on simvastatin, a class $1 \mathrm{~A}$ medication, and fenofibrate developed acute pancreatitis. In their report, there was no rechallenge as the patient expired during a complicated hospital course.

Our patient was on atorvastatin, a class III medication, for many years without complications. He developed acute pancreatitis subsequent to the addition of fenofibrate six months prior to his initial presentation. The patient developed a recurrence of pancreatitis within 72 hours of fenofibrate rechallenge. In addition, the patient had a prior cholecystectomy and an EUS was negative for biliary stones, strictures, dilations, sludge, and chronic pancreatitis. A $12 \mathrm{~mm}$ x $20 \mathrm{~mm}$ pancreatic mass was found without pancreatic duct stenosis, strictures, or dilation and FNA revealed no malignant cells. Given that all other known causes of acute pancreatitis were ruled out, along with a positive rechallenge to the medication, we theorize that fenofibrate was a probable cause of recurrent drug-induced pancreatitis. Also, it is possible that the combination of fenofibrate and statin use can increase the risk for the disease. Furthermore, we believe that fenofibrate may produce pancreatitis by a different mechanism than previously theorized in literature, as there was no evidence of biliary stones or sludge in this case.

\section{Conclusion}

This case identifies fenofibrate as a probable cause of druginduced pancreatitis, in addition to highlighting the importance of medication review in patients presenting with recurrent disease. We hypothesize that the mechanism of action regarding fenofibrate-induced pancreatitis is separate from the previously stated mechanism of lithogenic potential of other lipid lowering agents. It is also possible that the combination of fenofibrate with a statin can increase the risk of recurrent drug-induced pancreatitis. 


\section{Conflicts of Interest}

The authors declare that they have no conflicts of interest.

\section{References}

[1] K. B. McDonald, B. G. Garber, and M. M. Perreault, "Pancreatitis associated with simvastatin plus fenofibrate," Annals of Pharmacotherapy, vol. 36, no. 2, pp. 275-279, 2002.

[2] P. Somani, T. Sunkara, and M. Sharma, "Role of endoscopic ultrasound in idiopathic pancreatitis," World Journal of Gastroenterology, vol. 23, no. 38, pp. 6952-6961, 2017.

[3] R. D. Bowers, S. M. Valanejad, and A. A. Holombo, "Mirtazapine-induced pancreatitis-a case report," Journal of Pharmacy Practice, 2018.

[4] M. R. Jones et al., "Drug-induced acute pancreatitis: a review," The Ochsner Journal, vol. 15, pp. 45-51, 2015.

[5] N. Badalov, R. Baradarian, K. Iswara, J. Li, W. Steinberg, and S. Tenner, "Drug-induced acute pancreatitis: an evidence-based review," Clinical Gastroenterology and Hepatology, vol. 5, no. 6, pp. 648.e3-661.e3, 2007.

[6] W. Y. Hung, "Contemporary review of drug-induced pancreatitis: A different perspective," World Journal of Gastrointestinal Pathophysiology, vol. 5, no. 4, p. 405, 2014.

[7] The Coronary Drug Project Research Group, "Gallbladder disease as a side effect of drugs influencing lipid metabolism experience in the coronary drug project," The New England Journal of Medicine, vol. 296, no. 21, pp. 1185-1190, 1977.

[8] D. Preiss, M. J. Tikkanen, P. Welsh et al., "Lipid-modifying therapies and risk of pancreatitis: A meta-analysis," Journal of the American Medical Association, vol. 308, no. 8, pp. 804-811, 2012.

[9] N. Gang, P. Langevitz, and A. Livneh, "Relapsing acute pancreatitis induced by re-exposure to the cholesterol lowering agent bezafibrate," American Journal of Gastroenterology, vol. 94, no. 12, pp. 3626-3628, 1999.

[10] M. Miettinen, O. Turpeinen, and M. J. Karvonen, "A cooperative trial in the primary prevention of ischaemic heart disease using clofibrate.," British Heart Journal, vol. 42, no. 3, pp. 370-371, 1979. 